\title{
A Proposed Approach for Efficient Energy Utilization in Cloud Data Center
}

\author{
Akhil Goyal \\ Centre for Development of Advanced Computing \\ (C-DAC), Mohali
}

\author{
Navdeep S. Chahal \\ Centre for Development of Advanced Computing \\ (C-DAC), Mohali
}

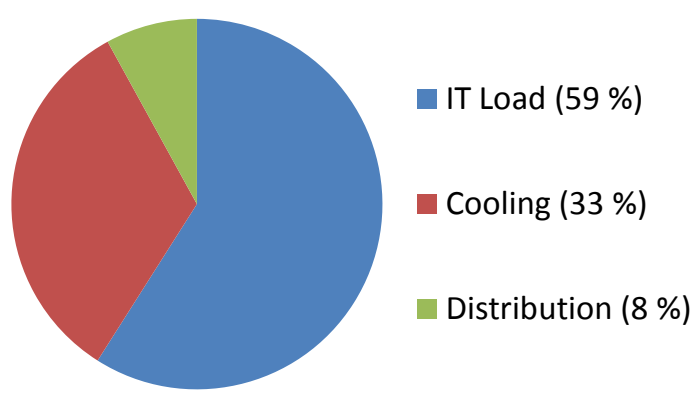

\begin{abstract}
Cloud computing has transmuted the IT industry on the whole and made easy to access the resources from anywhere and at anytime. These resources can be accessed as a service rather than as a product. Clients pay for the service used as per their requirement. The huge demand of cloud computing has given rise to the creation of hefty amount of data centers. These data centers consist of different types of resources and each resource is bound to its own power consumption scenario. Besides a number of benefits; Cloud data centers also pose the problem of large amount of power consumption. Lots of techniques and methods have been developed to solve this issue out of which load balancing is one such technique. This paper discusses load balancing using a nature inspired algorithm known as Particle Swarm Optimization (PSO) algorithm. A different nature inspired algorithm known as Firefly Algorithm (FA) is proposed to solve the above said issue.
\end{abstract}

\section{General Terms}

Resource Utilization, bio-inspired algorithms, meta-heuristic algorithms, Resource allocation, Resource Provisioning, Energy saving techniques

\section{Keywords}

Cloud Computing, load balancing, Energy Consumption, PSO Algorithm, Firefly Algorithm, Cloud Data center.

\section{INTRODUCTION}

Cloud Computing is a collection of different technologies in which very huge amount of infrastructure participates. This infrastructure contains a large number of computers which are accessed through the internet. Cloud Computing brings revolution in Information and Communication Technology (ICT) industry where user can get resources as per their requirement and accordingly pay for it [3]. Every organization has its different resource requirements. Also these requirements are not fixed throughout and may vary frequently. These dynamic requirements can be fulfilled with the help of cloud computing technology. Even organization having low start-up investment can avail the services of high cost computing infrastructures. These services may be in the form of infrastructure which includes the physical computers or virtual machines etc., or it can be services related to application software's and services related to Operating systems, Databases, and web servers etc. This revolution in cloud computing gave birth to the establishment of hefty amount of Data Centers. These data centers contain huge amount of nodes for computation and results in the consumption of lots of electrical energy. 
This research is also focused on the same above mentioned scenario. In this aspect Ashwin et. al. [3] has implemented the Particle Swarm Optimization Load Balancing Algorithm and calculated the response time. In this paper Firefly (FA) algorithm is proposed to perform load balancing in cloud data center and the energy consumed is evaluated for the same. Rest of this paper is organized as follows: Section 2 details the Related Work in this field. Section 3 explains the algorithm (Particle Swarm Optimization) already implemented for load balancing by Ashwin et al. through which the proposed research is motivated. The proposed approach is discussed in Section 4. Paper is concluded and summarized in Section 5.

\section{RELATED WORK}

As already stated lot of work has been done in this field. Pushtikant Malviya et al. [9] presented a very efficient method of resource provisioning to reduce the power consumption in virtual cloud environment. The researcher tried various energy saving techniques such as Dynamic Voltage Frequency Scaling Technique (DVFS), Allocation of various resources or Migration of Virtual Machine Techniques in different practical conditions to make the cloud environmental friendly. The main focus of his research was to do effective resource provisioning based on adaptive migration of VM's.

Wanneng Shu et al. [12] presented a new energy effective algorithm for green cloud computing in which the resources are allocated on the basis of immune clonal optimization method. The performance of the algorithm was analyzed on cloudsim tool kit. The algorithm is based on three models: time, cost and energy consumption. In experimental results it was found that the response time and make span was significantly improved so the above approach has a huge potential to improve energy efficiency in cloud data centers.

R. Yamini et al. [10] implemented green algorithm for power management in cloud computing. To streamline the resource usage in cloud computing task consolidation is needed which in turn increases the energy efficiency. It is a fact that with the increase of resource utilization the energy consumption can be decreased keeping this in mind the author successfully modeled the relationship between these two parameters and designed two energy-conscious task consolidation heuristics. The author also included the cost parameter in these heuristics which is used to calculate the energy saving potential and further verified.

Anton Beloglazov et al. [2] presented the efficient way to manage the data centers for cloud computing. This paper describes the heuristic developed for the energy aware resource allocation. In their research work they proposed architectural principles for energy-efficient management for cloud. They also proposed the techniques and scheduling algorithms for energy effective resource distribution keeping in mind the QoS and power usage characteristics.

Akhil et al. [1] focused on various soft computing techniques for load balancing. These approaches are very efficient for solving NP-complete problems and load balancing is considered as NP-complete problem. This paper describes comparisons between various soft computing techniques by using some fixed parameters and specifies the advantages and disadvantages among them.

Kumar Nishant et al. [6] have implemented a modified Ant Colony Optimization (ACO) Algorithm for the purpose of load balancing suitable for both cloud and grid computing environment. In contrast to regular ACO, in this modified algorithm each ant updates its own result set after each iteration rather than updating the result set given by head ant. The key criterion for load balancing in this algorithm is based on the aspect that each available resource is to be utilized to its fullest.

Yanwen Xiao et al [15] implemented a heuristic algorithm for data placement. In this paper the author focuses on node scheduling to minimize energy consumption in cloud. The main objective of this research is to accommodate maximum data on minimum number of nodes. Author has named the above said objective as set cover problem and the problem is successfully resolved using Greedy approach. This approach is further simulated practically using cloudsim.

Jens Buysse et al. [5] proposed a two step approach to reduce the energy consumption in optical clouds. In this paper a routing and scheduling algorithm is implemented, which selects unused nodes and turnoff these nodes. Scheduling focuses on the selection of source and destination node in one step and routing decides a unicast route from that particular source to a particular destination in second step. The results show that energy consumption during scheduling and routing using algorithm is $50 \%$ less as compared to traditional scheduling and routing.

Bharti Wadhwa et al. [4] done a review on different approaches to save energy consumption in green cloud computing. This paper describes the hard work of different researchers to minimize the carbon footprints emissions by techniques used for virtualization, VM migration and scheduling.

An-Ping Xiong et al. [7] focused on three basic goals to minimize energy consumption in cloud environment. Either the quality of service can be reduced to a reasonable extent to minimize energy consumption, or fix a maximum quantitative value for energy and then extract maximum performance in that definite amount, or negotiate the energy and performance parameters effectively and efficiently so that both are not compromised. The author pursue with third criteria with the help of virtualization technology to perform VM consolidation using PSO algorithm. The results show that energy is minimized but the algorithm is restricted to only two instances (CPU and Disk). The other resources such as network resources, memory etc is not taken into account.

\section{PARTICLE SWARM OPTIMIZATION ALGORITHM (PSO)}

Energy consumption is one of the major issue in cloud computing. As already discussed, there is numerous energy saving techniques available to minimize energy consumption in cloud data centers. Load Balancing is among one such technique. By reviewing the above mentioned approaches it was also found that meta-heuristic algorithms are proved best to solve the various NP-complete problems and load balancing also comes under the same category [14].

Ashwin et al. [3] has done load balancing using Particle Swarm Optimization Algorithm (PSO). By using PSO algorithm, the author performed resource allocation of virtual machines on the basis of upcoming user requests. The load was distributed in a very effective way among the available machines. Target was to compute the response time and keep that as small as possible. The whole experiment is performed practically using Cloud Analyst simulator. Final results are compared with Modified Throttled algorithm. 


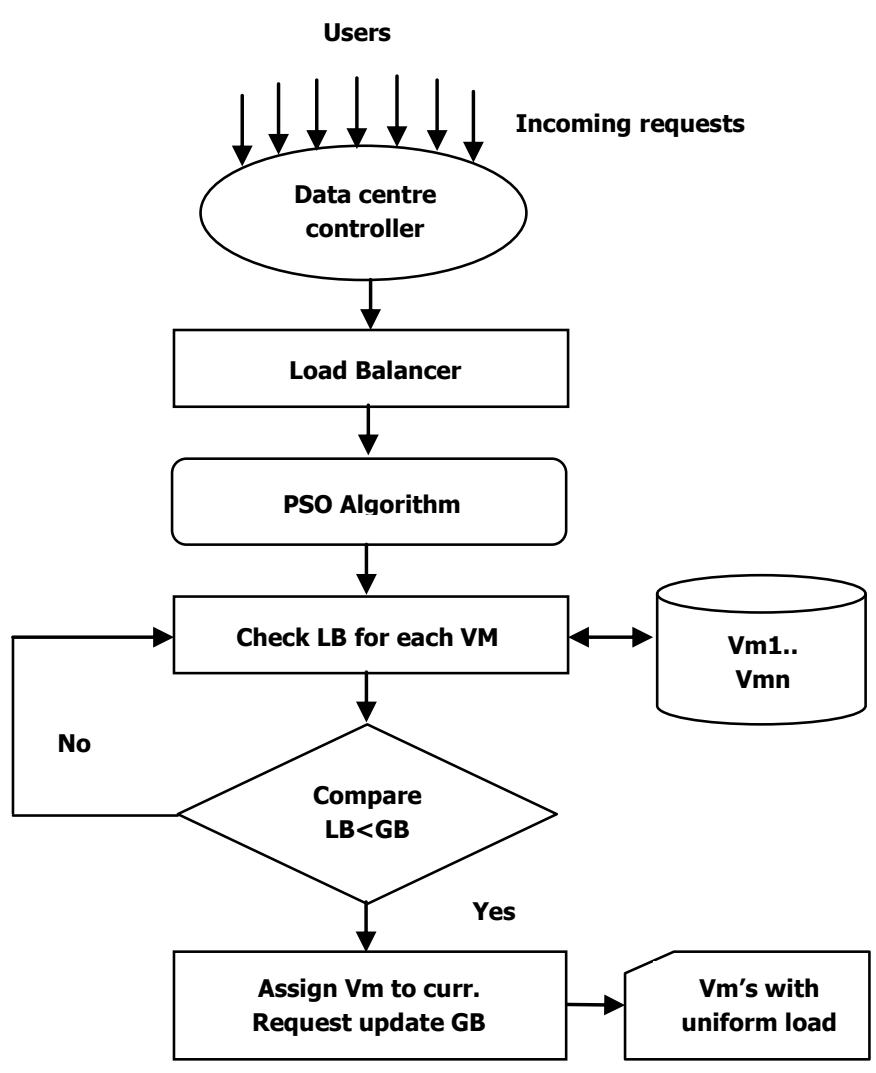

Fig 2: Working of PSO Algorithm

PSO algorithm is encouraged by the behavior of bird flocking and fish flocking in the manner they search for their food in the search space. While searching their food in the search space every entity in the flock moves randomly and changes its direction, scatters and regroups until they find the food. Every single entity in flock is considered as a particle which is associated with a velocity and position vectors. The value of each particle is calculated by some fitness function. The two main parameters which are considered in the evaluation of PSO are Pbest and Gbest. Pbest is defined as the best position of the particular particle in the entire flock and Gbest is the best fitness value obtained by the particle in the entire flock.

PSO algorithm is an iterative algorithm. The Global best position in PSO is obtained by iteratively improving Local best position to a given threshold value. Initially all the VMs are set to a same status that is available. Some load is already there with each VM. That load value is stored in a variable VMcount. This VMcount will become the Local Best parameter for each VM and can vary after each iteration. This Local Best is to be compared Global Best which decides whether the load is to be allocated to this particular VM or not. A VM having less Local Best value will get the load and in second case load will have to wait till the criteria matches.

\section{PROPOSED ALGORITHM}

Proposed approach is a bio inspired approach from swarm intelligence category that focuses on the working behavior of natural fireflies. FA was originally developed by Xin-She Yang at Cambridge University in 2007. Natural fireflies exhibit a flashing behavior that encompasses three basic rules.

- Fireflies are unisex species so each firefly can be attracted to any other firefly.

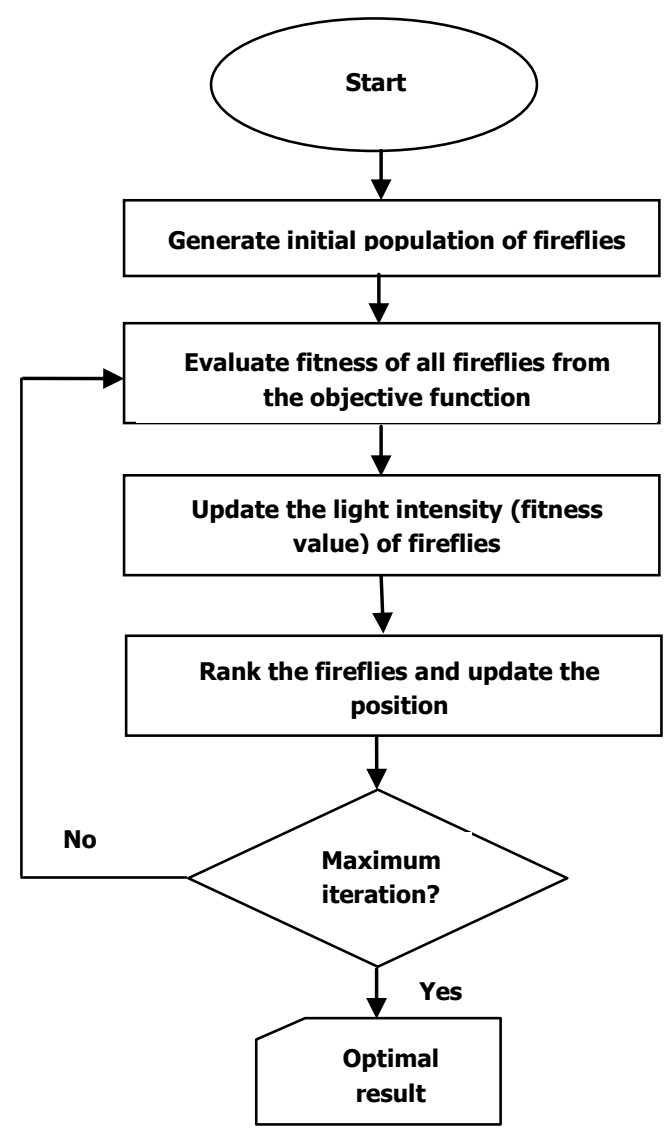

Fig 3: Flowchart for Firefly Algorithm

- This attraction is controlled by the aspect that the firefly having less brightness magnitude will be attracted towards the firefly with brightness magnitude

- If the magnitude of brightness is same for both the fireflies pairing up; each firefly can move randomly in its search space.

As described in Flowchart in Figure 3; to compute this brightness a fitness function is used and this brightness is related to the distance in terms of inverse square law; that is Brightness (I) $\alpha 1 /$ Distance $(\mathrm{R})^{2}$. Distance between the two fireflies is computed as the Cartesian distance. As the gap amongst the two fireflies increases, the corresponding brightness magnitude reduces proportionally (according to inverse square law). In the proposed Firefly Algorithm (FA) the brightness function is assumed as a load function. Less bright Firefly is assumed as under loaded node. When brightness magnitude is more, the node is considered as having load more than underutilized node but it can be less than or equal to threshold value. A threshold value is perceived for maximum load that can be handled by a particular virtual node at any time. Any node at any time should not have load more than that threshold value. Our motive is to take all the nodes to that threshold level.

Moreover load balancing helps to balance the load among all the available nodes which increases the resource utilization and hence, saves the energy consumed by the nodes. Even a server which is not in use or in little use consumes $70 \%$ of energy of their peak power [13].

Firefly Algorithm is considered better than other natureinspired optimization algorithms in the sense that it is simple 
to implement, having less complexity. Control is not centralized at a single point thus is more scalable. Related to Load Balancing in Cloud computing environment, Firefly has already been implemented for scheduling, and clustering scenarios.

\section{CONCLUSION AND FUTURE WORK}

In this paper, various energy efficient methods are studied which have been proved very important to reduce energy consumption in cloud computing. This paper also discusses the Particle Swarm Optimization Algorithm (PSO) for load balancing in cloud data center and also a new Firefly Algorithm (FA) is proposed. According to related work, it has been concluded that Firefly Algorithm should give better results as compared to PSO [11]. In future work we will implement the proposed algorithm on cloud analyst toolkit and Java programming. The algorithm can also be further compared with the PSO algorithm to know whether the results match with our assumptions or not.

\section{REFERENCES}

[1] Akhil Goyal, Bharti, "A Study of Load Balancing in Cloud Computing using soft Computing Techniques," International Journal of Computer Applications (c) 2014 by IJCA Journal, Vol. 92, No. 9, 2014.

[2] Anton Beloglozov, Jemal Abawajy, Rajkumar Buyya, "Energy-aware Resource Allocation Heuristics for Efficient management of Data centers for Cloud Computing," Future Generation Computer Systems, vol. 28, pp. 755-768, ELSEVIER 2012.

[3] Ashwin T.S., Shridhar G. Domanal and G. Ram Mohana Reddy, "A Novel Bio-Inspired Load Balancing of Virtual Machines in Cloud Environment," in IEEE conference, Banglore, pp. 1-4, Oct, 2014.

[4] Bharti Wadhwa, Amandeep Verma, "Energy Saving approaches for Green Cloud Computing: A Review," Proceedings of RAECS UIET Panjab University Chandigarh, IEEE, 06 - 08 March, 2014.

[5] Jens Buysse, Konstantinos Georgakilas, Anna Tzanakaki, Marc De Leenheer, Bart Dhoedt, Chris Develder, "Energy- Efficient Resource-Provisioning Algorithm for Optical Clouds," Journal of Optical Communication Network, Vol. 5, No. 3, pp. 226-239, March 2013.

[6] Kumar Nishant, Pratik Sharma, Vishal Krishna, Chhavi Gupta, Kunwar Pratap Singh, Nitin, Ravi Rastogi, "Load Balancing of Nodes in Cloud using Ant Colony
Optimization," 14th International Conference on Modelling and Simulation, IEEE, pp. 3-8, 2012.

[7] An-ping Xiong, Chun-xiang Xu, "Energy Efficient Multiresource Allocation of Virtual Machine Based on PSO in Cloud Data Center," Hindawi Publishing Corporation, Mathematical Problems in Engineering, Vol. 2014, Article ID 816518, June, 2014.

[8] http://www.koomey.com; Last accessed on Jonathan Koomey, Ph.D. Research fellow, Steyer-Taylor Center for Energy Policy and Finance, Stanford University, Presented at The Physics Of Sustainable Energy III University of California, Berkeley, March 82014.

[9] Pushtikant Malviya, Swapnamukta Agrawal, Shailendra Singh, "An Effective Approach for Allocating VMs to Reduce the Power Consumption of Virtualized Cloud Environment," Fourth International Conference on Communication Systems and Network Technologies, IEEE, 2014

[10] R. Yamini, "Power Management in Cloud Computing Using Green Algorithm," IEEE-International Conference on Advances in Engineering, Science and Management (ICAESM 2012) pp. 128-133, March 30, 31, 2012

[11] Saibal K. Pal, C.S. Rai, Amritpal Singh, "Comparative Study of Firefly Algorithm and Particle Swarm Optimization for Noisy Non-Linear Optimization Problems," I.J. Intelligent Systems and Applications, pp. 50-57, 2012

[12] Wanneng Shu, Wei Wang, Yunji Wang, "A Novel Energy-Efficient Resource Allocation Algorithm based on Immune Clonal Optimization for Green Cloud Computing," EURASIP Journal on Wireless Communications and Networking, Springer, 2014.

[13] X. Fan, W.D. Weber, L.A. Barroso, "Power Provisioning for Warehouse-Sized Computer," in proceedings of the 34th International Symposium on Computer Architecture (ISCA), pp. 13-23, 2007.

[14] X.S. Yang, "Nature-Inspired Meta-heuristic Algorithms," Luniver Press, 2010.

[15] Yanwen Xiao, Jinbao Wang, Yaping li, Hong Gao, "An Energy-Efficient Data Placement Algorithm and Node Scheduling Strategies in Cloud Computing Systems," 2nd International Conference on Advances in Computer Science and Engineering, Atlantis Press (CSE 2013). 